\title{
SÍNDROME CARDIORRENAL: FISIOPATOLOGIA E TRATAMENTO
}

Samuel de Paula Miranda', Rafael Nogueira de Macedo', Geraldo Bezerra da Silva Júnior², Elizabeth De Francesco Daher*3

Trabalho realizado pelo Serviço de Nefrologia, Hospital Universitário Walter Cantídio, Faculdade de Medicina, Universidade Federal do Ceará, Fortaleza,CE

*Correspondência: Rua Vicente Linhares, 1198 CEP 60270-135

Fortaleza - Ceará Tel / Fax: (85) 3224-9725 / (85) 3261-3777 ef.daher@uol.com.br/ geraldobezerrair@yahoo.com.br

\section{RESUMO}

A doença renal crônica (DRC) é um dos principais problemas de saúde pública mundial. A anemia é um frequente achado na DRC, afetando aproximadamente $90 \%$ dos pacientes. A relação entre anemia e doença cardiovascular já é bastante estabelecida e resulta de alterações na estrutura do ventrículo esquerdo e sua função. A anemia per se pode induzir significante morbidade cardíaca na ausência de doença renal e é um fator de risco independente para complicações cardíacas em paciente com insuficiência renal. O presente artigo de revisão avalia as inter-relações entre anemia, insuficiência cardíaca e doença renal, incluídas recentemente na chamada síndrome da anemia cardiorrenal.

UnITERMOS: Anemia. Insuficiência cardíaca. Insuficiência renal.

\section{INTRODUÇÃo}

A doença renal crônica (DRC) é um dos principais problemas de saúde pública mundial, apresentando altas taxas de morbi-mortalidade, principalmente quando associada à insuficiência cardíaca congestiva (ICC)!

As doenças cardiovasculares são as principais causas de morbidade e mortalidade em pacientes submetidos a tratamento dialítico, contando com quase $40 \%$ das hospitalizações e aproximadamente $50 \%$ dos óbitos ${ }^{2}$. A taxa de mortalidade cardiovascular em pacientes com doença renal em fase terminal (DRT) é I0-20 vezes maior que na população em geral, sendo a taxa de sobrevida em cinco anos estimada em aproximadamente 20\% (Figura I).

Uma avaliação detalhada das comorbidades em 822 pacientes que iniciaram tratamento dialítico revelou que a ICC estava presente em mais de um terço dos casos, e que era um preditor de morte prematura ${ }^{4}$. A gravidade da ICC correlaciona-se também com o risco de morte precoce (Figura 2).

Os determinantes de doença cardiovascular (DCV) na população em terapia de reposição renal (TRR) são múltiplos e incluem os fatores de risco identificados na populaçãa em geral e os fatores de risco adicionais específicos da DRC 5 , 6 .

A anemia afeta cerca de $90 \%$ dos pacientes com $\mathrm{DRC}^{7}$. A relação entre anemia e DCV já é bastante estabelecida e resulta de alterações na estrutura do ventrículo esquerdo (VE) e sua função. Essas alterações levam a uma hipertrofia ventricular esquerda (HVE) adaptativa, evoluindo para uma cardiomiopatia descompensada, que predispõe à ICC, doença cardíaca isquêmica e, em última instância, à morte prematura ${ }^{3,8}$.

\section{Figura I - Taxa de sobrevida de homens (a) e mulheres (b) acima de 64 anos de idade (adaptado de Parfrey et al. ${ }^{3}$ )}

a)

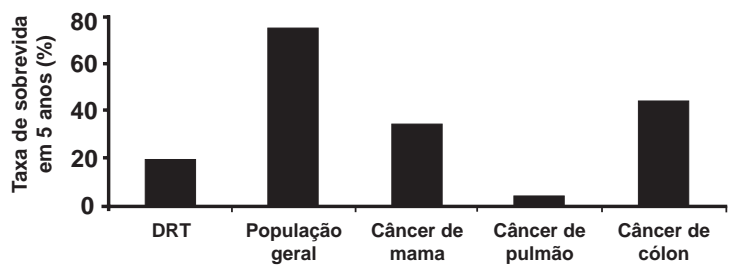

b)

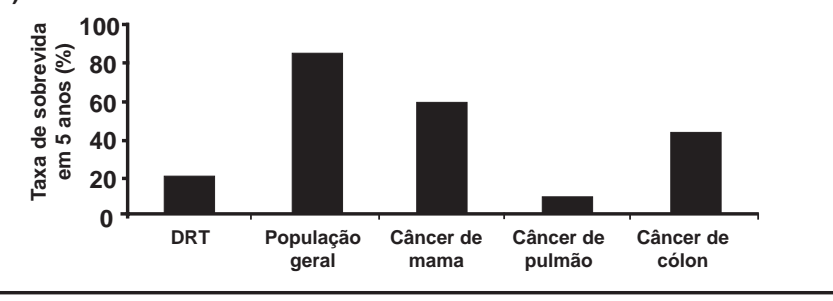

No caso da anemia, a taxa de evidência atualmente sugere que: (I) per se ela pode induzir significante morbidade cardíaca na ausência de doença renal; (II) é um fator de risco independente para complicações cardíacas em pacientes com insuficiência renal; (III) a correção com eritropoetina (EPO) pode aumentar o aporte de oxigênio para o coração e reverter as mudanças patológicas na geometria do VE?.

\footnotetext{
1. Acadêmico de Medicina, Universidade Federal do Ceará. Fortaleza, Brasil

2. Residente de Nefrologia do Hospital Universitário Walter Cantídio, Universidade Federal do Ceará. Fortaleza, Brasil

3. Professora Adjunta da Disciplina de Nefrologia, Departamento de Medicina Clínica, Universidade Federal do Ceará. Fortaleza, Brasil
} 


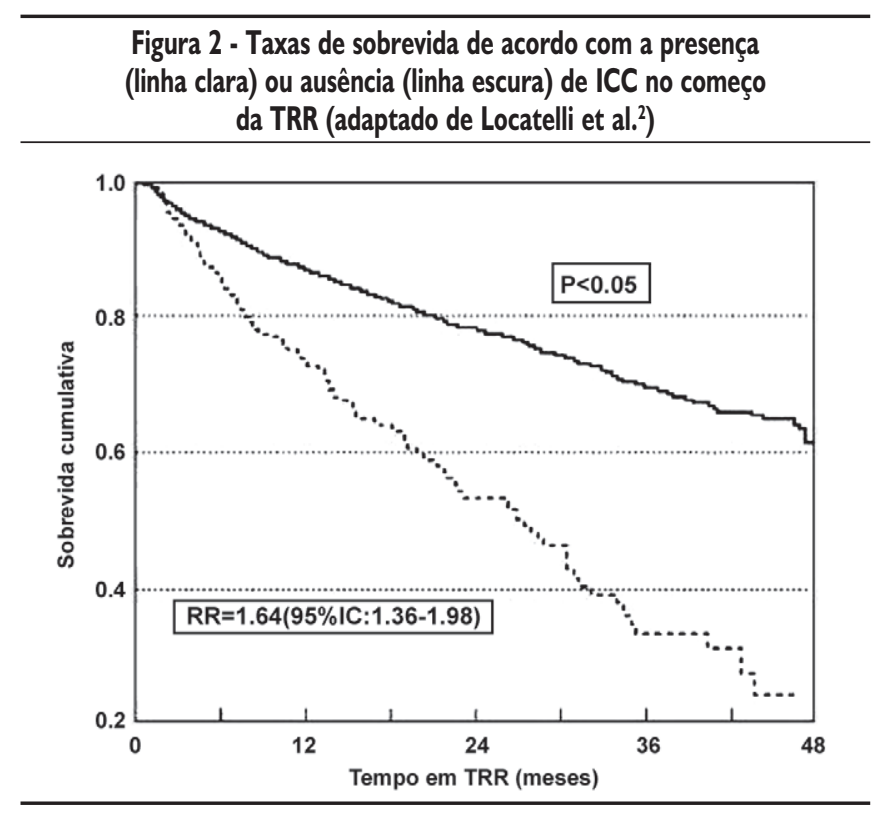

\section{A anemia na doença renal crônica}

Os primeiros sinais de anemia na DRC tornam-se evidentes quando a taxa de filtração glomerular (TFG) cai para abaixo de $50 \mathrm{~mL} / \mathrm{min}$. Concentrações de hemoglobina $(\mathrm{Hb})$ são $2,5 \mathrm{~g} / \mathrm{dL}$ menores em pacientes com TFG de $50-25 \mathrm{~mL} / \mathrm{min}^{10}$. A eritropoiese encontra-se diminuída na DRC, devido tanto aos efeitos das toxinas retidas sobre a medula óssea como à diminuição da síntese de eritropoetina pelos rins lesados ou à presença de inibidores da eritropoetina. $\mathrm{O}$ uso de eritropoetina recombinante humana determina uma elevação notável do hematócrito e da hemoglobina"l.

Outras situações podem contribuir para o advento de anemia em pacientes portadores de DRC, como deficiência de ferro, ácido fólico e vitamina B I2; perdas sanguíneas, hemólise e inflamação". A perda de sangue é excessiva em pacientes submetidos à hemodiálise, devido à necessidade de heparina durante o processo dialítico ${ }^{12}$. Outro mecanismo seria a correlação entre citocinas pró-inflamatórias e anemia. Foram propostos alguns mecanismos, incluindo:

\section{Supressão da eritropoese na medula óssea}

Provavelmente, é o mecanismo mais importante para a anemia induzida por citocina pró-inflamatória. As citocinas IL-I, TNF- $\alpha$ e IFN-Y têm demonstrado efeitos supressores sobre a eritropoiese, e este mecanismo provavelmente é o mais importante na patogênese da anemia em pacientes com inflamação ${ }^{13}$. Além disso, IL-I e IL-6 podem antagonizar a habilidade da eritropoetina de estimular a proliferação da medula óssea em cultura ${ }^{14}$. Em um estudo com 90 pacientes em hemodiálise seguidos por mil dias ${ }^{15}$, a IL-6 foi o mais forte preditor de morte.

\section{Citocinas podem inibir a produção de eritropoetina}

Pacientes com anemia da doença crônica têm níveis inapropriadamente baixos de eritropoetina para o seu grau de anemia ${ }^{16}$, e tem sido postulado que citocinas pró-inflamatórias podem causar anemia ao inibir a secreção de eritropoetina. Foi demonstrado em estudos experimentais que $|L-| \alpha,|L-| \beta$ e TNF- $\alpha$ inibem a produção de eritropoetina ${ }^{17}$.

\section{Sangramento intestinal}

Outro mecanismo em potencial para a anemia devido a citocinas pró-inflamatórias é o sangramento aumentado pela parede intestinal. Um estudo holandês demonstrou significante perda sanguínea intestinal em ratos tratados com injeções de IL-6 por via intraperitoneal por 14 dias, comparado com o grupo controle ${ }^{18}$. Estudos in vivo têm demonstrado que injeções intravasculares de rh-TNF- $\alpha$ podem levar à diarréia aquosa, síndrome do escape vascular e necrose de vilosidades, bem como a uma resposta inflamatória aguda com hemorragia de ceco ${ }^{19}$.

\section{A influência da anemia na insuficiência cardíaca congestiva}

Silveberg et al. ${ }^{20}$ publicaram observações referentes à alta prevalência e severidade da anemia na ICC. Em 142 casos de ICC acompanhados em um ambulatório de cardiologia, 55,6\% tinham anemia, definida como $\mathrm{Hb}<12 \mathrm{~g} / \mathrm{dl}$. Quanto mais severa a ICC mais prevalente e severa era o grau de anemia do paciente. Nos pacientes com ICC mais severa (Classe IV da New York Heart Association NYHA), 79, I\% eram anêmicos ${ }^{21}$. Em estudo recente realizado em nossa região, observou-se anemia em $45 \%$ de 174 pacientes internados com insuficiência cardíaca, e $82 \%$ apresentavam algum grau de insuficiência renal22.

Uma recente análise de dados do Canadian Organ Replacement Registry enfatiza a correlação entre anemia e mortalidade em pacientes dialíticos. Para ambos pacientes em hemodiálise (HD) e diálise peritoneal (PD), baixos níveis de hemoglobina $(\mathrm{Hb})$ foram associados com aumento de mortalidade ${ }^{23}$. No estudo de Silva et al., ${ }^{22}$ a anemia não esteve associada à mortalidade, entretanto a presença de disfunção renal (evidenciada pela elevação da creatinina sérica) esteve associada a um maior risco de óbito $(\mathrm{OR}=1,5, \mathrm{IC} 95 \%=1,0-2,3, \mathrm{p}=0,02)^{22}$. Um nível baixo de $\mathrm{Hb}$ tem sido identificado como um fator de risco independente para ICC em pacientes dialíticos ${ }^{24}$. Além disso, a anemia é associada com a presença de dilataçãao de VE no começo da diálise e subsequente crescimento do $\mathrm{VE}^{25,26}$.

Silveberg et al. ${ }^{21}$ realizaram uma metanálise de 44 estudos que examinavam a relação entre a anemia, gravidade da ICC, hospitalização e mortalidade. Dos 17 estudos que analisavam a relação entre a severidade da ICC e da anemia, 13 deles (76,5\%) revelaram que a presença de anemia era associada com um grau mais severo de ICC de acordo com a classe funcional NYHA. Dos 29 estudos que analisavam a relação entre mortalidade e ICC, 28 estudos (96,6\%), exceto um ${ }^{27}$, mostraram que a presença de anemia era associada a altos níveis de mortalidade. Em vários desses estudos as relações entre anemia e ICC permaneceram estatisticamente significantes após análise multivariada, na qual a função renal e a idade também eram levadas em consideração, ou seja, a anemia era um fator de risco independente para mortalidade cardíaca. Foi demonstrado em alguns desses estudos que insuficiência renal e anemia têm efeito aditivo na mortalidade ${ }^{28,29}$. 


\section{Fisiopatologia da injúria cardíaca pela anemia}

A anemia, quando suficientemente severa, pode causar insuficiência cardíaca até em indivíduos normais ${ }^{30}$ (Figura 3). Um estudo recente com mais de um milhão de pacientes idosos mostrou que a anemia era um preditor independente do desenvolvimento de ICC ${ }^{31}$.

Existem vários mecanismos pelos quais a anemia pode levar à ICC, entre eles:

\section{Aumento da retenção hídrica, da atividade simpática e da atividade} do sistema renina-angiotensina-aldosterona (SRAA)

A hipóxia tecidual e vasodilatação periférica presentes na anemia causam diminuição da pressão sanguínea arteria ${ }^{30}$, levando a um aumento da resposta simpática, a qual leva à taquicardia, aumento do volume corrente, vasoconstrição e redução do fluxo sanguíneo renal e, finalmente, à retenção de sal e água. O fluxo sanguíneo renal reduzido ativa o SRAA e o hormônio antidiurético $(A D H)$, causando vasoconstricção renal, aumentando mais ainda a retenção hidrossalina. Isso leva a um edema periférico e um aumento do volume plasmático. O volume plasmático aumentado causa sobrecarga ventricular, levando a um posterior estresse na parede do miocárdio. Sob a influência do SRAA e da atividade simpática aumentada, ocorre a hipertrofia ventricular esquerda, o que resulta em morte da célula miocárdica por necrose e apoptose ${ }^{32,33}$. Isso pode levar ou piorar a ICC.

\section{Hipóxia cardíaca direta}

A falta de suprimento de oxigênio para o coração, em face à frequência cardíaca e volume corrente elevados (trabalho cardíaco aumentado), leva à dilatação ventricular, hipertrofia, morte da célula miocárdica, fibrose cardíaca e ICC ${ }^{34}$.

\section{Estresse oxidativo aumentado}

Eritrócitos contêm muitos antioxidantes. A anemia é, portanto, associada ao aumento do estresse oxidativo ${ }^{35}$, o que causa dano às células miocárdicas ${ }^{34}$.

\section{Influência da concentração de hemoglobina sobre o sistema cardiovascular}

O oxigênio distribuído para um órgão ou tecido é proporcional a três fatores: (I) fatores hemodinâmicos, como débito cardíaco e a sua distribuição; (II) capacidade carreadora de oxigênio do sangue, como a concentração de hemoglobina e (III) extração de oxigênio, como a diferença de saturação de oxigênio entre sangue arterial e venoso ${ }^{36}$. Mecanismos hemodinâmicos e não-hemodinâmicos operam para compensar a diminuição da concentração de hemoglobina. Mecanismos não-hemodinâmicos incluem produção aumentada de eritropoetina para estimular eritropoiese e extração aumentada de oxigênio. Este último é alcançado pela diminuiç̧ão da afinidade entre oxigênio e hemoglobina, o que desloca a curva de dissociação hemoglobinaoxigênio para a direita. Em repouso, os fatores não-hemodinâmicos e a diminuição da afinidade entre oxigênio e hemoglobina podem, quase completamente, compensar o déficit de hemoglobina, limitando o papel dos fatores hemodinâmicos ${ }^{37}$. Dessa maneira, o débito cardíaco

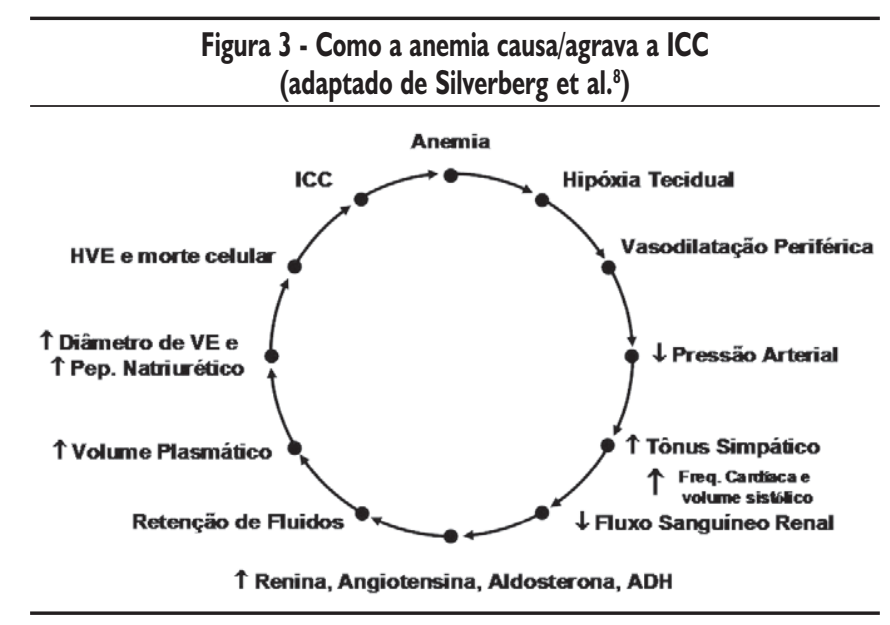

em repouso só aumenta quando a concentração de hemoglobina cai para $\mathrm{log} / \mathrm{dL}$ ou menos $^{38}$.

Quando a concentração de hemoglobina está abaixo de $10 \mathrm{~g} / \mathrm{dL}$, ou durante condições de não-repouso, o débito cardíaco e fluxo sanguíneo aumentados começam a compensar a hipóxia tecidual, superpondo-se aos fatores não-hemodinâmicos. Os mecanismos de compensação hemodinâmica são variados e complexos, destacando-se: (I) redução na sobrecarga devido à diminuição da resistência vascular sistêmica, (II) aumento na pré-carga devido ao aumento do retorno venoso e (III) aumento da função do ventrículo esquerdo (VE) atribuída ao aumento da atividade simpática e a fatores inotrópicos ${ }^{39}$.

A diminuição da resistência vascular é uma consequência direta da redução da viscosidade sanguínea e dilatação arterial. O decréscimo na viscosidade sanguínea é devido à diminuição do numero de eritrócitos e redução do hematócrito, o que caracteriza a anemia. A dilatação arterial é devido à dilatação vascular stricto sensu, resultante do aumento do diâmetro arteriolar, mas também envolve o recrutamento de novos vasos, formação de colaterais e shunts arteriovenosos ${ }^{40}$. Alguns mecanismos foram propostos, incluindo: (I) "vasodilatação hipóxica" devido a metabólitos gerados pela hipóxia e (II) "vasodilatação fluxo-mediada" devido ao aumento do fluxo sanguíneo, um efeito que é mediado por células endoteliais e fatores relaxantes derivado do endotélio ${ }^{4 !}$.

$\mathrm{O}$ aumento na pré-carga resulta do aumento do retorno venoso, o que ocorre por conta da diminuição do retorno venoso. A resistência ao retorno venoso diminui em paralelo com o decréscimo do hematócrito e da viscosidade sanguínea. Além disso, a vasodilatação arteriolar facilita a transmissão de pressão do sistema arterial para a circulação venosa, criando um gradiente de pressão favorável ao retorno venoso. Finalmente, o aumento da atividade simpática pode induzir uma venoconstricção ativa, favorecendo o enchimento cardíaco ${ }^{42}$.

$\mathrm{Na}$ anemia crônica, sobrecargas de fluxo/volume de longa duração e o trabalho cardíaco aumentado levam ao crescimento progressivo e à $H V E^{43}$. Em pacientes em DRT, as alterações de função e estrutura cardíacas são acompanhadas paralelamente por remodelamento dos condutos arteriais maiores ${ }^{44}$. 


\section{Anemia e hipertrofia ventricular esquerda na doença renal crônica}

London et al. ${ }^{45}$ demonstraram que existe uma associação entre anemia da DRT e o desenvolvimento de HVE. Concentrações de Hb correlacionam-se tanto com volume diastólico final do VE quanto com a massa do VE. Parece haver dois tipos de pacientes: um cuja anemia resulta em um aumento exclusivo da massa do VE e outro cuja anemia também resulta em dilatação do volume diastólico final do VE.

A anemia da DRT também tem importantes efeitos sobre os grandes vasos. London et al. ${ }^{46}$ mostraram que o diâmetro da artéria carótida comum é significativamente aumentado em pacientes com DRT. A área de secção e a espessura do músculo medial também são aumentadas, enquanto a distensibilidade muscular é reduzida. Quando essas alterações são combinadas com o aumento da velocidade do fluxo que é vista caracteristicamente em pacientes anêmicos com DRC, existe um risco aumentado para aterosclerose pré-matura. A combinação de anemia com HVE é relacionada com riscos cardíacos aumentados ${ }^{47}$.

Um estudo canadense multicêntrico prospectivo, com 446 pacientes com taxas de clearance de creatinina estimadas variando de 25 - 75mL/min encontrou que 34\% da coorte desenvolveram HVE no decorrer do estudo, com uma prevalência máxima entre aqueles com pior função renal| ${ }^{48}$. Uma análise dos fatores de risco para esta ICC de novo identificou níveis baixos de $\mathrm{Hb}$ juntamente com idade avançada e pressão arterial sistólica elevadaa ${ }^{49}$.

\section{Tratamento da anemia em pacientes com DRC}

O tratamento da anemia é recomendado para todos os pacientes com DRC por já ter sido demonstrados os efeitos benéficos não apenas sobre o estado cardiovascular ${ }^{50}$, mas também sobre a qualidade de vida do paciente $^{5 !}$.

\section{Hematócrito e concentração de hemoglobina recomendados para pacientes com DRC}

A concentração de Hb ótima objetivada para pacientes com DRC em terapia com EPO ainda não foi completamente estabelecida, apesar da publicação de guidelines nos Estados Unidos e na Europa. Os US National Kidney Foundation - Dialysis Outcomes Quality Iniciative (NKF - DOQI) guidelines, inicialmente publicados em 1997, recomendam um hematócrito alvo entre $33 \%$ e $36 \%$ ou uma concentração de $\mathrm{Hb}$ entre $1 \mathrm{e}$ e $12 \mathrm{~g} / \mathrm{dL}^{52}$. A resposta à EPO é determinada primariamente pela disponibilidade de ferro ${ }^{53,54}$.

Em pacientes com DRC, o suprimento de ferro para a formação de novos eritrócitos é diminuído, devido tanto à perda sanguínea quanto à absorção reduzida. Um estudo demonstrou que aproximadamente três litros de sangue podem ser perdidos por ano e $2400 \mathrm{~mL}$ durante procedimentos de diálise ${ }^{55}$.

O uso apropriado da suplementação de ferro é um ponto chave para o sucesso da terapia com EPO. Ferro intravenoso é mais efetivo que o ferro oral em aumentar a eritropoese e reduzir a dose de EPO requerida para pacientes em hemodiálise ${ }^{56}$, e a rota intravenosa já está atualmente bem estabelecida como via de administração de ferro em pacientes desse grupo ${ }^{57}$.
Os critérios para reposição de ferro são: saturação de transferrina $<20 \%$ e/ou ferritina sérica $<100 \mathrm{~g} / \mathrm{mL}$. O ferro pode ser reposto inicialmente via oral, porém muitos pacientes terão que receber o ferro por via endovenosa, pois o ferro por via oral pode ocasionar intolerância gástrica e nem sempre é absorvido em quantidade suficiente para repor de maneira adequada os estoques do paciente. $\mathrm{O}$ emprego de ferro intravenoso é bem tolerado, porém é recomendado que seja feito com supervisão médica ou de enfermagem $^{58}$. Muitas vezes é verificada a correção da anemia apenas com a reposição de ferro, prescindindo, portanto, do emprego de eritropoetina ${ }^{59}$.

Existem evidências demonstrando que o emprego de eritropoetina melhora significativamente a qualidade de vida de pacientes com doença renal crônica no período pré-dialítico ${ }^{60}$.

Vários estudos realizados em pacientes portadores de DRC em tratamento conservador mostraram que o emprego de EPO em pacientes com DRC pré-dialítica é eficaz na correção da anemia e não acelera a perda de função renal residual e em alguns deles observou-se inclusive desaceleração da progressão da DRT ${ }^{61,62}$.

A eritropoetina pode ser administrada por via endovenosa (sem diluir, I a 2 minutos) ou subcutânea. A via subcutânea é a via preferencial para uma maior eficiência da medicação ${ }^{63}$. A dose inicial é de 80-160 U/ $\mathrm{Kg} / \mathrm{semana}$, dividida em uma ou duas injeções por semana. Esta dose inicial deverá ser ajustada de acordo com a variação dos níveis de hematócrito e hemoglobina.

\section{Conflito de interesse: não há}

\section{SUMMARY}

Cardio-renal syndrome: PATHOPHYSIOLOGY and tREATMENT

Chronic kidney disease (CKD) is one of the most important problems of public health, worldwide. Anemia is frequent in CKD, affecting approximately $90 \%$ of patients. The relationship between anemia and cardiovascular disease is well established and is due to alterations in left ventricular structure and function. Anemia per se can lead to significant cardiac morbidity in the absence of renal disease and is an independent risk factor for cardiac complication in the patient with renal insufficiency. The present review evaluates the inter-relations between anemia, heart failure and renal disease, which were recently included in the so-called cardio-renal anemia syndrome. [Rev Assoc Med Bras 2009; 55(I): 89-94]

KEY wORDS: Anemia. Heart failure. Renal failure.

\section{RefERÊNCIAS}

I. Cleland JGF, Khand A, Clark AL. The heart failure epidemic: exactly how big is-it? Eur Heart J. $2001 ; 22(5): 623-6$.

2. Locatelli F, Marcelli D, Conte F. Cardiovascular disease in chronic renal failure: the challenge continues. Registro Lombardo Dialisi e Trapianto. Nephrol Dial Transplant. 2000; I 5(Suppl 5):69-80.

3. Parfrey P. Anaemia in chronic renal disease: lessons learned since Seville 1994. Nephrol Dial Transplant. 2001 ; I 6(Suppl 7):4 I-5.

4. Barrett BJ, Parfrey PS, Morgan J, Barre P, Fine A, Goldstein MB, et al. Prediction of early death in end-stage renal disease patients starting dialysis. Am J Kidney Dis. 1997;29(2):214-22. 
5. Luke RG. Chronic renal failure: a vasculopathic state. N Eng J Med. 1998;339(I2):84|-3.

6. Locatelli F, Bommer J, London GM, Martin-Malo A, Wanner C, Yaqoob $M$, et al. Cardiovascular disease determinants in chronic renal failure: clinical approach and treatment. Nephrol Dial Transplant. 2001; 16(3):459-68.

7. Tsakiris D. Morbidity and mortality reduction associated with the use of erythropoietin. Nephron. 2000;85(Suppl I):2-8.

8. Silverberg DS, Wexler D, laina A, Schwartz D. The interaction between heart failure and other heart diseases, renal failure, and anemia. Semin Nephrol. 2006;26(4):296-306

9. Eckardt KU. Cardiovascular consequences of renal anaemia and erythropoietin therapy. Nephrol Dial Transplant. 1999; | 4(5): I 3 | 7-23.

10. Zoccali C. Cardiorenal risk as a new frontier of nephrology: research needs and areas for intervention. Nephrol Dial Transplant. 2002; I 7(Suppl I I):50-4.

II. Hutchinson FN, Jones WJ. A cost-effectiveness analysis of anemia screening before erythropoietin in patients with end-stage renal disease. Am J Kidney Dis. 1997;29(5):65I-7.

12. Skorecki K, Green J, Brenner BM. Insuficiência renal crônica. In: Kasper DL, Fauci AS, Longo DL, Braunwald E, Hauser SL, Jameson JL, editores. Harrison medicina interna. $16^{a}$ ed. Rio de Janeiro: McGraw-Hill Interamericana do Brasil; 2006. p. 1732-42.

13. Macdougall I. Role of uremic Toxins in exacerbating anaemia in renal failure. Kidney Int. 200 I;59(Suppl):S67-S72.

14. Schooley JC, Kullgren B, Allison AC. Inhibition by interleukin-I of the action of erythropoietin on erythroid precursors and its possible role in the pathogenesis of hypoplastic anaemias. Br J Haematol. 1987;67( I): I I 7.

15. Stenvinkel P. The role of inflammation in the anaemia of end-stage renal disease. Nephrol Dial Transplant. 200 I; I6 (Suppl 7):36-40.

16. Miller CB, Jones RJ, Piantadosi S, Abeloff MD, Spivak JL. Decreased erythropoietin response in patients with the anemia of cancer. $\mathrm{N}$ Engl J Med. 1990;322(24): 1689-92.

17. Jelkmann W, Pagel H, Wolff M, Fandrey J. Monokines inhibiting erythropoietin production in human hepatoma cultures and in isolated perfused rat kidneys. Life Sci. 1992;50(4):30 I-8.

18. Jongen-Lavrencic M, Peeters HR, Rozemuller H, Rombouts WJ, Martens AC, Vreugdenhil G, et al.. IL-6-induced anaemia in rats: possible pathogenetic implications for anemia observed in chronic inflammations. Clin Exp Immunol. 1996; 103(2):328-34.

19. Patton JS, Peters PM, McCabe J, Crase D, Hansen S, Chen AB, et al. Development of partial tolerance to the gastrointestinal effects of high doses of recombinant tumor necrosis factor-alpha in rodents. J Clin Invest. 1987;80(6): 1587-96

20. Silverberg DS, Wexler D, Blum M, Keren G, Sheps D, Leibovitch E, et al. The use of subcutaneous erythropoietin and intravenous iron for the treatment of the anemia of severe, resistant congestive heart failure improves cardiac and renal function, functional cardiac class, and markedly reduces hospitalization. J Am Coll Cardiol. 2000;35(7): 1737-44.

21. Silverberg $D S$, Wexler $D$, laina $A$. The role of anemia in the progression of congestive heart failure. Is there a place for erythropoietin and intravenous iron? J Nephrol. 2004; 17(7):749-6I.

22. Silva RP, Barbosa PH, Kimura OS, Sobrinho CR, Sousa Neto JD, et al. Prevalence of anemia and its association with cardio-renal syndrome. Int J Cardiol. 2007; I 20(2):232-6.

23. Murphy SW, Barrett BJ, Kent GM, et al. Anemia and mortality in peritoneal dialysis patients. J Am Soc Nephrol. 2000; I I (2):290A.

24. Harnett JD, Foley RN, Kent GM, Barre PE, Murray D, Parfrey PS. Congestive heart failure in dialysis patients: prevalence, incidence, prognosis and risk factors. Kidney Int. 1995;47(3):884-90.

25. Foley RN, Parfrey PS, Kent GM, Harnett JD, Murray DC, Barre PE. Longterm evolution of cardiomyopathy in dialysis patients. Kidney Int. 1998;54(5): 1720-5.

26. Parfrey PS, Foley RN, Harnett JD, Kent GM, Murray DC, Barre PE. Outcome and risk factors for left ventricular disorders in chronic uraemia. Nephrol Dial Transplant. 1996; I (7): |277-85.
27. Tanner H, Moschovitis G, Kuster GM, Hullin R, Pfiiffner D, Hess OM, et al. The prevalence of anemia in chronic heart failure. Int J Cardiol. 2002;86(I): II5-21.

28. McClellan WM, Flanders WD, Langston RD, Jurkovitz C, Presley R. Anemia and renal insufficiency are independent risk factors for death among patients with congestive heart failure admitted to community hospitals: a population-based study. J Am Soc Nephrol. 2002; I3(7): 1928-36.

29. Gregory DD, Sarnak MJ, Konstam MA, Pereira B, Salem D. Impact of chronic kidney disease and anemia on hospitalization expense in patients with left ventricular dysfunction. Am J Cardiol. 2003;92( I I): I300-5.

30. Anand IS, Chandrashekhar Y, Ferrari R, Poole-Wilson PA, Harris PC. Pathogenesis of edema in chronic anemia: Studies of body water and sodium, renal function, haemodynamics and plasma hormones. Br Heart J. 1993;70(4):357-62.

31. Sandgren E, Murray AM, Herzog CA. Anemia and new-onset congestive heart failure in the general Medicare population. J Cardiac Fail. 2005; I I ( I):99- I05.

32. Johnson DB, Dell Italia LJ. Cardiac hypertrophy and failure in hypertension. Curr Opin Nephrol Hypertens. 1996;5(2): | 86-9|.

33. Katz AM. The cardiomyopathy of overload: an unnatural growth response in the hypertrophied heart. Ann Intern Med. 1994; I 2 I (5):363-7|.

34. Silverberg D, Wexler D, Blum M, Wollman Y, laina A. The cardio-renal anaemia syndrome: does it exist? Nephrol Dial Transplant. 2003; | 8(Suppl 8):7- 12.

35. Siems WG, Sommerburg $O$, Grune T. Erythrocyte free radical and energy metabolism. Clin Nephrol. 2000;53(Suppl I):S9- 17.

36. Rosenthal DS, Braunwald E. Hematological-oncological disorders and heart disease. In: Braunwald E, editor. Heart disease: a textbook of cardiovascular medicine. $4^{\text {th }}$ ed. Philadelphia: WB Saunders; 1992.

37. Oski FA, Marshall BE, Cohen PJ, Sugerman HJ, Miller LD. The role of the left-shifted or right-shifted oxygen-hemoglobin equilibrium curve. Ann Intern Med. 1971;74(I):44-6.

38. Varat MA, Adolph RJ, Fowler NO. Cardiovascular effects of anemia. Am Heart J. 1972;83(3):4 I 5-26.

39. Muller R, Steffen HM, Brunner R, Saric J, Pollok M, Baldamus CA, et al. Changes in the alpha adrenergic system and increase in blood pressure with recombinant human erythropoietin (rHuEpo) therapy for renal anemia. Clin Invest Med. 199|;|4(6):6|4-22.

40. Martin C, Yu AY, Jiang BH, Davis L, Kimberly D, Hohimer AR, et al. Cardiac hypertrophy in chronically anemic fetal sheep: Increased vascularization is associated with increased myocardial expression of vascular endothelial growth factor and hypoxia-inducible factor. Am J Obstet Gynecol. 1998; 178(3):527-34.

4I. Anand IS, Chandrashekhar Y, Wander GS, Chawla LS. Endotheliumderived relaxing factor is important in mediating the high output state in chronic severe anemia. J Am Coll Cardiol. 1995;25( I I): | 402-7.

42. Metivier F, Marchais SJ, Guerin AP, Pannier B, London GM. Pathophysiology of anaemia: focus on the heart and blood vessels. Nephrol Dial Transplant. 2000; I 5(Suppl 3): |4-8.

43. London GM, Parfrey PS. Cardiac disease in chronic uremia: pathogenesis. Adv Ren Replace Ther. 1997;4(3): 194-21 I.

44. London GM, Guerin AP, Marchais SJ, Pannier B, Safar ME, Day M, et al. Cardiac and arterial interactions in end-stage renal disease. Kidney Int 1996;50(2):600-8.

45. London GM, Marchais SJ, Guerin AP, Fabiani F, Metivier F. Cardiovascular function in hemodialysis patients. Adv Nephrol Necker Hosp. 1991;20(2):249-73.

46. London GM, Marchais SJ, Guerin AP, Metivier F, Pannier B. Cardiac Hypertrophy and arterial alterations in end-stage renal disease: hemodinamic factors. Kidney Int Suppl. 1993;4I (I):S42-S9.

47. Mann JF. What are the short-term and long-term consequences of anaemia in CRF patients? Nephrol Dial Transplant. 1999;।4(Suppl 2):29-36.

48. Foley RN, Parfrey PS. Cardiac disease in chronic uremia: clinical outcome and risk factors. Adv Ren Replace Ther. 1997;4(3):234-48. 
49. Rigatto C, Jeffrey J, Foley RN. Risk factors for de novo congestive heart failure (CHF) in renal transplant recipients. J Am Soc Nephrol. 2000; I I (7):705A.

50. Hayashi T, Suzuki A, Shoji T. Cardiovascular effect of normalizing the hematocrit level during erythropoietin therapy in predialysis patients with chronic renal failure. Am J Kidney Dis. 2000;35(2):250-6.

5I. Moreno F, Sanz-Guajardo D, Lopez-Gomez JM, Jofre R, Valderrabano F, for the Spanish Cooperative Renal Patients Quality of Life Study Group of the Spanish Society of Nephrology. Increasing the hematocrit has a beneficial effect on quality of life and is safe in selected hemodialysis patients. J Am Soc Nephrol. 2000; I I (4):335-42.

52. NKF-DOQI clinical practice guidelines for the treatment of anemia of chronic renal failure. National Kidney Foundation-Dialysis Outcomes Quality Initiative. Am J Kidney Dis. 1997;30(Suppl 3): 192-240.

53. Macdougall IC. Poor response to erythropoietin: practical guidelines on investigation and management. Nephrol Dial Transplant. 1995; 10(5):607- 14.

54. Horl WH. Is there a role for adjuvant therapy in patients being treated with epoetin? Nephrol Dial Transplant. 1999; I 4(Suppl 2):50-60.

55. Moore LW, Acchiardo S, Sargent JA, Burk L. Incidence, causes and treatment of iron deficiency anaemia in hemodialysis patients. J Renal Nutr. 1992;3(1): 105-12.

56. Fishbane S, Frei GL, Maesaka J. Reduction in recombinant human erythropoietin doses by the use of chronic intravenous iron supplementation. Am J Kidney Dis. 1995;26(I):41-6.

57. Macdougall IC, Hutton RD, Cavil I, Coles GA, Williams JD. Poor response to treatment of renal anaemia with erythropoietin corrected by iron given intravenously. Br Med J. 1989;299(??): I57-8.
58. Abensur H. Anemia da doença renal crônica. J Bras Nefrol. 2004;26(Supl I):26-8.

59. Silverberg DS, Blum M, Agbaria Z, Deutsch V, Irony M, Schwartz D, et al. The effect of i.v. iron alone or in combination with low-dose erythropoietin in the rapid correction of anemia of chronic renal failure in the predialysis period. Clin Nephrol. 200 1;55(3):212-9.

60. The US Recombinant Human Erythropoietin Predialysis Study Group. Double-blind, placebo-controlled study of the therapeutic use of recombinant human erythropoietin for anemia associated with chronic renal failure in predialysis patients. Am J Kidney Dis. I99|; | 8(I):50-9.

6I. Roth D, Smith RD, Schulman G, Steinman TI, Hatch FE, Rudnick MR, et al. Effects of recombinant human erythropoietin on renal function in chronic renal failure predialysis patients. Am J Kidney Dis. 1994;24(5):777-84.

62. Kuriyama S, Tomonari H, Yoshida H, Hashimoto T, Kawaguchi Y, Sakai $O$. Reversal of anemia by erythropoietin therapy retards the progression of chronic renal failure, especially in nondiabetic patients. Nephron. 1997;77(2): 176-85.

63. Besarab A, Reyes CM, Hornberger J. Meta-analysis of subcutaneous versus intravenous epoetin in maintenance treatment of anemia in hemodialysis patients. Am J Kidney Dis. 2002;40(3):439-46.

Artigo recebido: 23/12/07 Aceito para publicação: 19/04/07 